\title{
D-Dimer assay as a non invasive test for the diagnosis of left atrial Thrombi in Indian patients with Rheumatic MS
}

\author{
*Rajappa $\mathrm{M}^{1 \#}$, Sunil Roy $\mathrm{TN}^{2 \#}$, Raj $\mathrm{A}^{3}$, Trehan $\mathrm{V}^{3}$, Mallika $\mathrm{V}^{4}$
}

1. Department of Biochemistry, Jawaharlal Institute of Postgraduate Medical Education and Research, Puducherry, India

2. Department of Cardiology, Belhoul Speciality Hospital, Dubai, United Arab Emirates

3. Department of Cardiology, G.B. Pant Hospital, New Delhi, India

4. Department of Biochemistry, G.B. Pant Hospital, New Delhi, India

\# Both authors contibuted equally for this study and therefore both qualify to be co-first authors.

\begin{abstract}
Background: Systemic embolism is a serious and sometime fatal complication of rheumatic MS.

Objective: We assessed the predictive power of D-Dimer level to predict occurrence of left atrial (LA) thrombi in patients with rheumatic mitral stenosis (MS).

Methods: D-dimer levels were analyzed for 24 patients with rheumatic MS with LA clot and 22 patients with rheumatic MS with no LA clot undergoing transeosophageal echocardiography. A level more than $4 \mu \mathrm{g} / \mathrm{ml}$ was taken as elevated to predict the presence of LA clot in the study groups.

Results: For a cut-off value of $4 \mu \mathrm{g} / \mathrm{ml}$, sensitivity was $66.67 \%$ and specificity $100 \%$ for prediction of LA clot and AUC 0.710. A cut-off value of less than $1 \mu \mathrm{g} / \mathrm{ml}$, sensitivity was $91.67 \%$ and $87.5 \%$ negative predictive value for ruling out presence of LA clot and AUC 0.721 .

Conclusions: A higher value of D-dimer can predict the possible presence of a LA clot and very low value can predict absence of clot in patients with rheumatic MS.

Key words: Rheumatic MS, Left atrial thrombus, D-Dimer levels, Transoesophageal echocardiography

African Health Sciences 2013; 13(3): 584 - 589 http://dx.doi.org/10.4314/ahs.v13i3.9
\end{abstract}

\section{Introduction}

Systemic embolism is a serious and sometime fatal complication of rheumatic MS. ${ }^{1}$ A large body of evidence indicates that patients with MS are at increased risk of developing left atrial thrombus and the associated thromboembolic complications. Part of this risk may be attributed to the hypercoagulable state associated with atrial fibrillation $(\mathrm{AF}),{ }^{2}$ but there have been studies that systemic coagulation activity is increased in MS per se. ${ }^{3-6}$. Transthoracic echocardiography (TTE), although easily performed, is an insensitive tool to detect clots because the left atrial appendage (LAA) cannot be easily visualized. Transesophageal echocardiography (TEE) is more

*Corresponding author:
Dr. Medha Rajappa
Assistant Professor
Department of Biochemistry
Jawaharlal Institute of Postgraduate Medical
Education and Research
Puducherry
India
Phone: +91-94863-98875
E-mail: linkmedha@gmail.com

useful to diagnose thrombus in the LAA. The importance of documenting the presence of LA thrombus clinically is highly relevant, as these patients who have clots are thus at a high risk of cardiac thromboembolism.

Measurement of fibrin D-dimer levels has been advocated as a useful clinical marker of thrombogenesis. ${ }^{7}$ The use of $\mathrm{D}$-dimer levels in the investigation and management pathway of venous thromboembolism is well established. ${ }^{8-9}$ This marker has a high sensitivity and specificity in excluding thromboembolism when a well-defined assay is used in the appropriate clinical setting. As is in the case of venous thromboembolism, a negative $\mathrm{D}$-dimer may be used to exclude atrial thrombus and perhaps to decide on the need for anticoagulation in patients with rheumatic mitral valve disease irrespective of the clinical risk factors which is less sensitive for clinical decision making.

Few studies in patients with rheumatic MS have focused on D-dimer levels for prediction of left atrial thrombus in patients undergoing balloon mitral valvuloplasty. ${ }^{10-11}$ Hence, we designed this prospective study to investigate these issues. Because 
D-dimers are of particular use in cardiovascular disease due to their role in detecting the presence of thrombus or clot, we hypothesize that increased Ddimer level in Indian patients with valvular heart disease suggest the presence of intra-cardiac clot and hence the need for anticoagulation and contraindicated for balloon mitral valvotomy irrespective of the clinical predictors of embolic risk. It can also be possible that D-dimer level can be used as guide on the intensity of anticoagulation needed to minimize thrombogenesis.

\section{Methods}

46 patients with symptomatic rheumatic MS undergoing transeosophageal echocardiography (TEE) before percutaneous transmitral commissurotomy (PTMC) from G.B.Pant Hospital, New Delhi, India constituted the study population. The study protocol was approved by the hospital ethics committee and all patients and controls voluntarily gave informed consent. Those with left atrial clot in TTE, suspected left atrial clot or where the LA appendage was not clear on TTE underwent a TEE before PTMC procedure. 24 patients with rheumatic MS with left atrial clot on TEE formed the study population (Group A). Control group include 22 patients with rheumatic MS with no left atrial clot on TEE (Group B). Patients with the following were excluded from the study: (a) patients with inadequate TEE examination, (b) patients with renal failure, (c) patients with hepatic impairment, (d) acute or chronic infection and (e) neoplastic disease. None of the patients in the study population had stroke or peripheral vascular disease.

Detailed clinical history was taken for all patients and clinical examination was performed in all patients and controls. (Groups A and B). Five ml of blood was collected from the study group as well as from the control group for the D-Dimer assay under standard conditions. Five $\mathrm{ml}$ of blood was collected into sodium citrate bottles and centrifuged at $3000 \mathrm{rpm}$ for 20 minutes to obtain plasma. D-Dimer levels were estimated from plasma, using commercially available ELISA kits from Gen Way Biotech, San Diego, California, USA. and expressed in $\mu \mathrm{g} / \mathrm{ml}^{13}$

A D-dimer level more than $4 \mu \mathrm{g} / \mathrm{ml}$ was considered as high and used to differentiate those with and without LA clot on TEE and to assess whether there is significant difference between two groups. The sensitivity, specificity, positive predictive value, negative predictive value, and accuracy of $\mathrm{D}$ Dimer assay are determined using presence of clot in TEE as a gold standard. A subgroup analysis included comparison of D-Dimer assay in patients with sinus rhythm and atrial fibrillation in the study group.

\section{Statistical analysis}

To summarise the data obtained, continuous variables were expressed as mean \pm standard deviation and discrete variables were expressed as a percentage. Statistical analyses were performed with SPSS version 13 (SPSS Inc). Continuous variables were compared by Student's t-test. The independent predictive role of embolic predictors were evaluated by multiple regression analysis of the baseline parameters. The sensitivity, specificity, positive predictive value, negative predictive value and accuracy of D-Dimer level for the presence of thrombus was calculated using standard formulas. Statistical significance was ascribed, when $\mathrm{p}<0.05$. The receiver operating characteristic (ROC) curves were constructed using STATA software.

\section{Results}

There were 24 patients in group A (with LA clot) and 22 patients in group B (control group).There were 7 males and 17 females in the test group and 8 males and 14 females in the control group. The mean age of the population was 33 years (15-60 years) in the test group and 26 years (18-42 years) in the control group. In group A 20 patients were in atrial fibrillation and 4 in sinus rhythm. In group B 15 patients were in sinus rhythm and 7 patients were in atrial fibrillation. The baseline characteristics of both the groups were given in table 1 .

The $\mathrm{D}$-dimer values were measured in ranges of $0.5-1,1-2,2-4,4-8$, and more than 8 $\mu \mathrm{g} / \mathrm{ml}$ in all these patients. The D-dimer levels were more than $4 \mu \mathrm{g} / \mathrm{ml}$ in 16 patients in group A. Eight patients had value less than $4 \mu \mathrm{g} / \mathrm{ml}$. All patients who had a D-Dimer value of more than $4 \mu \mathrm{g} / \mathrm{ml}$ had a clot in the LA. In the control group (group B), none of the patients had D-dimer level more than $8 \mu \mathrm{g} /$ $\mathrm{ml}$. All the patients had D-dimer level less than 4 $\mu \mathrm{g} / \mathrm{ml}$ and in 14 patients, the values were less than 1 $\mu \mathrm{g} / \mathrm{ml}$. Eight patients had value between 1 and 4 $\mu \mathrm{g} / \mathrm{ml}$. LA size, MVA, mean gradient, and Pulmonary artery pressures were not different in patients with and without LA clot. The range of DDimer levels in all the patients in the study population are given in table 2 . 
Table 1: Baseline characteristics of the study population

\begin{tabular}{llll}
\hline & Group A $(\mathbf{n}=\mathbf{2 4})$ & Group B $(\mathbf{n}=\mathbf{2 2})$ & p value \\
\hline Age $($ mean \pm SD) & $33 \pm 13.62$ & $26 \pm 7.12$ & \\
Sex M/F & $7 / 17$ & $8 / 14$ & \\
AF & 20 & 7 & \\
MVA & $0.875 \pm 0.113$ & $0.94 \pm 0.156$ & 0.11 \\
Mean gradient & $16 \pm 4.52$ & $14.86 \pm 3.68$ & 0.35 \\
LA size & $4.68 \pm 0.56$ & $4.42 \pm 0.49$ & 0.10 \\
PA pressure & $52.66 \pm 15.43$ & $52.21 \pm 17.64$ & 0.92 \\
\hline
\end{tabular}

Table 2: D- dimer levels in the different range in the study population

\begin{tabular}{llc}
\hline D-dimer levels & $\begin{array}{c}\text { Group A } \\
(\mathbf{n}=\mathbf{2 4})\end{array}$ & $\begin{array}{c}\text { Group B } \\
(\mathbf{n}=\mathbf{2 2})\end{array}$ \\
\hline $0.5-1 \mu \mathrm{g} / \mathrm{ml}$ & 2 & 14 \\
$1-2 \mu \mathrm{g} / \mathrm{ml}$ & 3 & 0 \\
$2-4 \mu \mathrm{g} / \mathrm{ml}$ & 3 & 8 \\
$4-8 \mu \mathrm{g} / \mathrm{ml}$ & 13 & 0 \\
$>8 \mu \mathrm{g} / \mathrm{ml}$ & 3 & 0 \\
\hline
\end{tabular}

Receiver operating characteristic (ROC) curve for D-dimer levels was plotted by applying logistic regression to determine the cut-off having the highest sensitivity as well as specificity (figures 1 and 2 respectively). The sensitivity, specificity, positive and negative predictive values were calculated for different cut-off values for D-Dimer levels. When a cut-off value of $4 \mu \mathrm{g} / \mathrm{ml}$ was taken, the sensitivity was $66.67 \%$ and specificity $100 \%$ for the prediction of LAA clot. A cut-off value of less than $1 \mu \mathrm{g} / \mathrm{ml}$ has high sensitivity of $91.67 \%$ and $87.5 \%$ negative predictive value for ruling out the presence of LAA clot. Area under ROC curve was 0.710 (95\% CI: 0.558 - 0.834 ) for a cut-off value of $4 \mu \mathrm{g} / \mathrm{ml}$ for the prediction of LAA clot and 0.721 ( $95 \%$ CI: 0.569 - 0.843) for a cut-off value of $1 \mu \mathrm{g} / \mathrm{ml}$ for ruling out the presence of LAA clot. The sensitivity, specificity, predictive values, and area under ROC were for different cut-off values of D-Dimer are given in table 3 and ROC curves for cut-off values of $4 \mu \mathrm{g} / \mathrm{ml}$ and $1 \mu \mathrm{g} / \mathrm{ml}$ are given in figures 1 and 2 .

Table 3 : Sensitivity, specificity, positive predictive value, negative predictive value for different cut-off values for D-Dimer levels

\begin{tabular}{llllccc}
\hline Cut-off & Sensitivity & Specificity & $\begin{array}{c}\text { Positive } \\
\text { PV }\end{array}$ & $\begin{array}{c}\text { Negative } \\
\text { PV }\end{array}$ & $\begin{array}{c}\text { Area under } \\
\text { ROC }\end{array}$ & 95 \% CI \\
\hline $4 \mu \mathrm{g} / \mathrm{ml}$ & $66.67 \%$ & $100 \%$ & $100 \%$ & $73.3 \%$ & 0.710 & $0.558-0.834(\mathrm{p}=0.003)$ \\
$2 \mu \mathrm{g} / \mathrm{ml}$ & $79.1 \%$ & $63.64 \%$ & $70.37 \%$ & $73.68 \%$ & 0.628 & $0.569-0.843(\mathrm{p}=0.12)$ \\
$1 \mu \mathrm{g} / \mathrm{ml}$ & $91.67 \%$ & $63.64 \%$ & 73.35 & $87.5 \%$ & 0.628 & $0.473-0.765(\mathrm{p}=0.01)$ \\
\hline
\end{tabular}

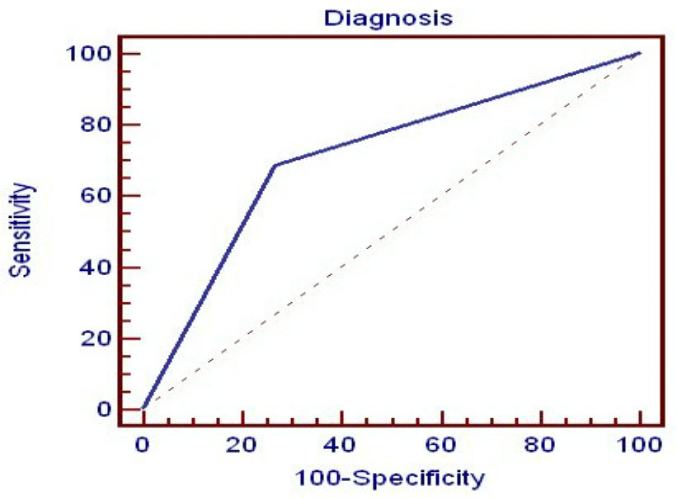

Figure 1: The sensitivity and Specificity and area under ROC for cut-off value for D-dimer level of $4 \mu \mathrm{g} / \mathrm{ml}$

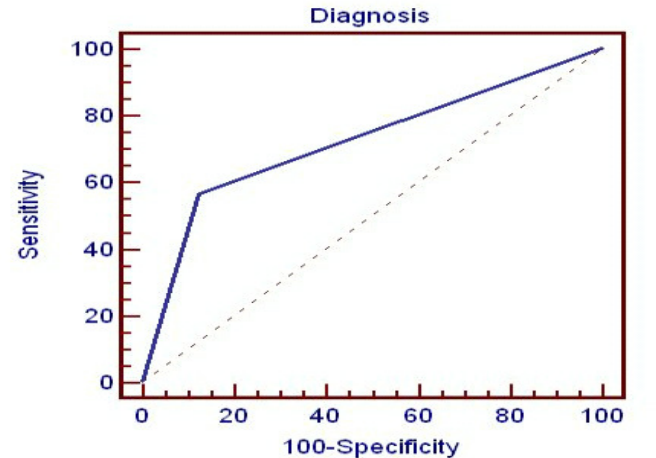

Figure 2: The sensitivity, specificty and area under ROC for cut-off level for D-dimer level of $1 \mu \mathrm{g} / \mathrm{ml}$

African Health Sciences Vol 13 Issue 3 September 2013 


\section{Discussion}

In the present study we analyzed D-dimer level as a non-invasive marker for the prediction of left atrial thrombus in North Indian patients with rheumatic MS undergoing PTMC. In the present study Ddimer levels were high in patients with left atrial appendage clot and a level more than $4 \mu \mathrm{g} / \mathrm{ml}$ has $100 \%$ specificity and positive predictive value for the presence of LAA clot. A value of less than 1 $\mu \mathrm{g} / \mathrm{ml}$ has very high sensitivity and negative predictive value for ruling out LAA clot in this group of patients.

Several clinical trials have indicated that patients with MS are at increased risk of devoloping left atrial thrombus and it may be attributed to the state of hypercoagulability associated with abnormalities of hemostasis, platelets and endothelial dysfunction. ${ }^{3-6}$. Having a marker of coagulation activation would be useful in identifying patients at highest thromboembolic risk, who might benefit most from anti-thrombotic therapy. Biomarkers of hypercoagulability, such as D-dimer, indicative of a prothrombotic state, can be used to predict those who are at increased risk of thromboembolism.

$\mathrm{D}$-dimers originate from the formation and lysis of cross-linked fibrin and are therefore specific markers of activation of coagulation. D-dimer levels may be increased as a result of co-morbid conditions causing intravascular or extravascular cross-linked fibrin turnover such as in renal failure, liver impairment, acute or chronic infection, neoplastic disease, hypertension, acute cardiovascular or cerebrovascular syndromes, bleeding, haematoma, and surgery. ${ }^{8}$ The interpretation of D-dimer levels can therefore be considered as reflecting the prothrombogenic state of patients without these acute clinical conditions and without overt thrombosis. Thrombus formation is a process involving platelets adherence to the vessel wall, platelet aggregation, thrombin generation and fibrin formation. As fibrin $\mathrm{D}$-Dimer is considered to originate from crosslinked fibrin assembled during thrombus formation, a rise in fibrin D-Dimer level is indicative of thrombus formation. ${ }^{8,9}$

Systemic thromboembolism is a serious complication in patients with valvular heart disease and its incidence is highest in those with MS. The status of fibrin generation and fibrinolysis in the peripheral blood of patients with MS has been studied by several groups. Yasaka et al ${ }^{10}$ reported that levels of fibrinopeptide A, D-Dimer, and antithrombin III in the peripheral blood were significantly higher in patients with MS than in normal subjects. Wang et al ${ }^{11}$ reported that patients with MS who were in sinus rhythm showed a significant increase in the D-Dimer level in the peripheral blood, when compared with control subjects; suggesting that the coagulation system is activated in patients with MS .

It has been suggested that the measurement of plasma fibrin D-Dimer concentration may be a useful screening method for patients at risk for intracardiac thrombi and hence thromboembolism. ${ }^{12,13}$ This was demonstrated in a prospective study of 63 patients with MS, in whom a significantly elevated plasma D-Dimer level was found in 10 patients with mobile intracardiac thrombus, when compared with patients with nonmobile thrombus or no thrombus. Umemeto et al ${ }^{14}$ examined the clotting and fibrinolytic activity in 37 patients with intracardiac thrombi. Plasma DDimer level was found to be positively related to clotting activity. If an excess of thrombosis over fibrinolysis was present, there was a substantial risk of arterial embolisation

Somoloi et al ${ }^{15}$ studied D-Dimer level in 75 patients undergoing electrical cardioversion in patients with atrial fibrillation and suggested that serum D-Dimer level is a useful marker of overall embolic risk in patients with atrial fibrillation and reflect a cumulative prothrombotic effect of clinical and echocardiographic predictors of stroke. Ddimer level as a single test adequately defined low thromboembolic risk associated with cardioversion of AF in two-third of patients. Habara et $\mathrm{a}^{16}$ measured D-dimer level in 925 patients with nonvalvular atrial fibrillation undergoing TEE, which detected 83 LAA thrombus and yielded a negative predictive value of $97 \%$ for identifying LAA thrombus for a cut-off value of $\mathrm{D}$-dimer. $\mathrm{D}$-dimer levels have been shown to be increased in AF especially in patients having multiple risk factors for embolism in earlier studies ${ }^{17,18}$. Ibebuogu et $\mathrm{al}^{18}$ described a case where elevated D-dimer resulted in performance of TEE, which documented atrial thrombus in a patient with infective endocarditis, atrial fibrillation and pulmonary edema and saved the patient from complications of cardioversion, which should have resulted from emergency cardioversion. Even the presence of a high D-dimer level is a strong predictor of survival in patients with chronic atrial fibrillation.

Low levels of D-dimer seen in patients with rheumatic MS with LA clot could be due to the 
effect of warfarin on reducing the levels of D-dimer as reported by some investigators early. ${ }^{19,20}$ In the present study, patients with rheumatic MS in our study were not on anti-coagulant (warfarin) therapy. If LA clot was detected by TTE/TEE in patients with rheumatic MS (Group A), D-dimer estimation was done first and then, the patient was started on anticoagulants, hence this would not affect their Ddimer levels. The control group (Group B) did not need anti-coagulant therapy.

\section{Study limitations}

Firstly, a small sample size was a major limitation in this study. Secondly, number of patients with atrial fibrillation were not comparable in the two groups. Patients with AF were higher in group A, compared to group B. Some elevation of D-dimer could be due to persistent atrial fibrillation and the elevation of $\mathrm{D}$-dimer in patients without LAA clot could also be due to patients with atrial fibrillation in this group. We included consecutive patients undergoing TEE before mitral valvuloplasty and our aim was to find out a non-invasive marker which could predict or rule out the presence of LA clot in this patients. If we had included patients with atrial fibrillation only, the interpretation should have been much easier, but the clinical utility of this test in an unselected patient group undergoing PTMC might have been less. However we need prospective validation of this different cut-off level of $\mathrm{D}$-dimer in predicting LA clot in consecutive patients undergoing TEE, in studies involving a larger study population, before widespread clinical use.

\section{Conclusion}

D-dimer levels were high in patients with left atrial appendage clot. In this group of patients, a level of D-dimer more than $4 \mu \mathrm{g} / \mathrm{ml}$ had 100\% specificity and positive predictive value for the presence of LAA clot. A value of less than $1 \mu \mathrm{g} / \mathrm{ml}$ had very high sensitivity and negative predictive value for ruling out LAA clot in this group of patients. Higher value of D-dimer could predict the possible presence of a LAA clot and very lower values of D-dimer could predict the possible absence of LAA clot in patients who were considered for PTMC, irrespective of the rhythm, LA size, mitral valve area, pulmonary artery pressure and mitral valve gradient. To the best of our knowledge, there are very few studies which demonstrate the above correlation in Indian patients with MS.
Patients with MS who were detected to have LA clot by TEE before PTMC, the serum level of D-dimer were higher, compared to MS patients without LA clot. All patients who had a D-Dimer value of more than $4 \mu \mathrm{g} / \mathrm{ml}$ had a clot in the LA, while none of the patients without clot had such a value. Majority of the patients without LA clot had D-dimer level less than $1 \mu \mathrm{g} / \mathrm{ml}$ and all the patients had value less than $4 \mu \mathrm{g} / \mathrm{ml}$. So a higher value of $\mathrm{D}$-dimer can be used to predict the possible presence of a clot and very low value of $\mathrm{D}$-dimer can be used to predict the absence of clot in patients with rheumatic MS, who are considered for PTMC, irrespective of the rhythm, LA size, mitral valve area, pulmonary artery pressure and mitral valve gradient.

\section{References}

1. Coulshed N, Epstein EJ, McKendrick CS, Galloway RW, Walker E. Systemic embolism in mitral valve disease. Br Heart J 1970; 32: 2634

2. Heppell R M, Berkin K E, McLenachan J M, Davies J A. Haemostatic and haemodynamic abnormalities associated with left atrial thrombosis in non-rheumatic atrial fibrillation. Heart. 1997;77: 407-11.

3. Yammaoto K, Ikeda U, Mito H, Shimada K et al Coagulation activity is increased in the left atrium of patients with MS. J Am Coll Cardiol 1995; 25: 107-12.

4. Li-Saw-Hee F L, Blann A D, Goldsmith I, Lip G Y. Indexes of Hypercoagulability Measured in Peripheral Blood Reflect Levels in Intracardiac Blood in Patients with Atrial Fibrillation Secondary to MS Am J Cardiol 1999;83:1206-1209

5. Boyaci A, Topaloglu S, Yilmaz S, Yanik O, Ozdemer O, Demer AD, Aras D, Kisacik H, Korkmaz S. Regional left trial coagulation and fibrinolytic activities in patients with MS. Jpn Heart J 2004; 45: 779-88

6. Ikeda U, Yamamoto K, Shimada K. Biochemical markers of coagulation activity in MS, atrial fibrillation and Cardiomyopathy. Clin Cardiol 1997: 20: 7-10

7. Shaun D Frost Rational use of D dimer measurment to exclude acute venous thromboembolic disease. Mayo Clinic Proce 2003; 78: 1385-91

8. Kelly J, Rudd A, Lewis R, Hunt BJ. Plasma Ddimer in the diagnosis of venous thromboembolism. Arch Intern Med 2002; 162: 
747-56

9. Lip G.Y, Lowe G.D. Fibrin D-dimer: A useful clinical marker of thrombogenesis? (editorial review) Medical Science 1995; 89: 205-14

10. Yasaka M, Miyatake K, Mitani M, Beppu S, Nagata S, Yamaguchi T, Omae T. Intracardiac mobile thrombus and D-dimer fragment of fibrin in patients with MS. Br Heart J 1991;66:22-25.

11. Wang J, Xie H, Huang J, Lu D, Yan Q. Hypercoagulability existing in the local left atrium of patient with MS. Chin Med J 2003; 116: $1198-202$

12. Tayebjee MH, LipGY. Fibrin D-dimer levels in atrial fibrillation as an index of thrombogenesis:A possible test to exclude left atrial thrombus? Am J Cardiol 2003; 92: 47-9

13. Vissac A, Grimaux M, Chartier S, Chan FA, Chambrette B, Amiral JV. A new sensitive membrane based ELISA technique for instantaneous D-dimer evaluation in Emergency. Thrombosis Research 1995; 78: 341 52

14 Yamamoto K, Ikeda U, Minezaki KK, Fukazawa H, Mizano O, Fujikawa H, Sekiguchi $\mathrm{H}$, Shimad K. Effect of mitral valvuloplasty in MS on coagulation activity Am J Cardiol 1997; 79: $1131-35$
15 Somoloi M, Tomcsanyi J, Nagy E, Bodo I, Bezzegh A. D-dimer determination as a screening tool to exclude atrial thrombi in atrial fibrillation. Am J Cardiol. 2003; 92:85-7.

16 Habara S,Dote K, Kato M, Sasaki S, Goto K, Takemoto H, Hasegawa D, Mastuda O. Predictor of left atrial appendage thrombi in non valvular atrial fibrillation. Eur Heart J 2007; 28: 2217-22.

17 Inoue H, Nozawa T, Okumura K, Lee JD, Shimuzu A, Yano K. Prothrombotic activity is icreased in patients with nonvalvular atrial fibrillation and risk factors for embolism. Chest 2004; 126: 687-92

18 Ibebuogu UN, Salah AK, Malhotra S, Calkins JB, Thornton JW, Mandawat M, Robinson VJB. The D-dimer assay: A possible tool in the evaluation of atrial thrombosis. Can J Cardiol 2008; 24: 517-9.

19 Mahe I, Drouet L, Simoneau G, Man-Muzeaux S, Caulin C, Bergmann J. D-dimer can predict survival in patients with chronic atrial fibrillation. Blood Coagul Fibrinolysis 2004; 15: 413-7

20 Perverill RE, Harris G, Gelman J Effect of Warfarin on regional left atrial coagulation activity in MS. Am J Cardiol 1997; 79: 339-43 